\title{
Liver imaging features by convolutional neural network to predict the metachronous liver metastasis in stage I-III colorectal cancer patients based on preoperative abdominal CT scan
}

Sangwoo Lee ${ }^{1 \dagger}$, Eun Kyung Choe ${ }^{2,3+}$, So Yeon Kim³ ${ }^{3,4}$, Hua Sun Kim ${ }^{5}$, Kyu Joo Park ${ }^{6+}$ and Dokyoon Kim ${ }^{3,7^{*+}+}$

From The 18th Asia Pacific Bioinformatics Conference

Seoul, Korea. 18-20 August 2020

\author{
* Correspondence: dokyoon.kim@ \\ pennmedicine.upenn.edu \\ 'Sangwoo Lee, Eun Kyung Choe, \\ Kyu Joo Park and Dokyoon Kim \\ contributed equally to this work. \\ ${ }^{3}$ Department of Biostatistics, \\ Epidemiology \& Informatics, \\ Perelman School of Medicine, \\ University of Pennsylvania, B304 \\ Richards Building, 3700 Hamilton \\ Walk, Philadelphia, PA 19104-6116, \\ USA \\ ${ }^{7}$ Institute for Biomedical Informatics, \\ University of Pennsylvania, \\ Philadelphia, PA 19104, USA \\ Full list of author information is \\ available at the end of the article
}

\begin{abstract}
Background: Introducing deep learning approach to medical images has rendered a large amount of un-decoded information into usage in clinical research. But mostly, it has been focusing on the performance of the prediction modeling for diseaserelated entity, but not on the clinical implication of the feature itself. Here we analyzed liver imaging features of abdominal CT images collected from 2019 patients with stage I - III colorectal cancer (CRC) using convolutional neural network (CNN) to elucidate its clinical implication in oncological perspectives.

Results: CNN generated imaging features from the liver parenchyma. Dimension reduction was done for the features by principal component analysis. We designed multiple prediction models for 5-year metachronous liver metastasis (5YLM) using combinations of clinical variables (age, sex, T stage, $\mathrm{N}$ stage) and top principal components (PCS), with logistic regression classification. The model using "1 ${ }^{\text {st }}$ PC (PC1) + clinical information" had the highest performance (mean AUC $=0.747$ ) to predict $5 Y L M$, compared to the model with clinical features alone (mean AUC $=0.709$ ). The PC1 was independently associated with 5YLM in multivariate analysis (beta $=-3.831, P<$ 0.001). For the 5 -year mortality rate, $P C 1$ did not contribute to an improvement to the model with clinical features alone. For the PC1, Kaplan-Meier plots showed a significant difference between PC1 low vs. high group. The 5YLM-free survival of low PC1 was 89.6\% and the high PC1 was 95.9\%. In addition, PC1 had a significant correlation with sex, body mass index, alcohol consumption, and fatty liver status.

(Continued on next page)
\end{abstract}

\section{$\triangle B M C$}

(c) The Author(s). 2020 Open Access This article is licensed under a Creative Commons Attribution 4.0 International License, which permits use, sharing, adaptation, distribution and reproduction in any medium or format, as long as you give appropriate credit to the original author(s) and the source, provide a link to the Creative Commons licence, and indicate if changes were made. The images or other third party material in this article are included in the article's Creative Commons licence, unless indicated otherwise in a credit line to the material. If material is not included in the article's Creative Commons licence and your intended use is not permitted by statutory regulation or exceeds the permitted use, you will need to obtain permission directly from the copyright holder. To view a copy of this licence, visit http://creativecommons.org/licenses/by/4.0/. The Creative Commons Public Domain Dedication waiver (http://creativecommons.org/publicdomain/zero/1.0/) applies to the data made available in this article, unless otherwise stated in a credit line to the data. 
(Continued from previous page)

Conclusion: The imaging features combined with clinical information improved the performance compared to the standardized prediction model using only clinical information. The liver imaging features generated by CNN may have the potential to predict liver metastasis. These results suggest that even though there were no liver metastasis during the primary colectomy, the features of liver imaging can impose characteristics that could be predictive for metachronous liver metastasis.

Keywords: Radiomics, Colorectal cancer, Convolutional neural network, Artificial intelligence

\section{Background}

In colorectal cancer (CRC) patients, liver is the most common site of metastasis due to its anatomical connection with portal circulation [1]. In advanced CRC, liver may be the sole site of metastasis in $30-40 \%$ of patients $[2,3]$. In these patients, median survival is $5-20$ months without intervention, and 5-year survival is extremely rare [4]. Thus, understanding the pathophysiology of the liver metastasis is one of the most critical subjects for CRC management.

Radiomics is an innovative technique that uses the large volume of imaging features to predict oncological features [5]. It consists of converting the medical images into high-dimensional features which is then used to predict clinical outcomes [6].Convolutional neural networks (CNNs) have recently manifested the ability to generate useful features from imaging data in various medical research [7]. There are multiple studies suggesting the CT imaging features extracted by CNNs have high predictive values in oncological outcomes $[8,9]$. Machine learning approach is one of the major subfields of artificial intelligence which can be used for constructing prediction model in radiomics [6] and has shown promising performances for predicting various oncological subjects [10-13].

Nonetheless, there are challenges that to evaluate the values of each imaging feature in the prediction model for an oncological outcome is difficult [14]. In traditional approach for radiological research, to review the medical images, radiologists use the human visual interpretation based on the characteristics of the images systematized by accumulated experiences and researches in association with clinical manifestations [15] and the statistical models are used to find associations in these data that could enhance clinical reasoning [16]. This canonical way to use medical images for clinical research might be presenting challenges to radiomics, which uses the imaging features generated by CNNs and designs prediction models by machine learning to enhance clinical performances. If the approaches of radiomics study can encompass purposes focusing on not only the performance of the prediction modeling for disease-related entity, but also the clinical implication of the imaging features itself, its utility and contribution to the healthcare research would be in great value.

Here we propose a framework to extract the liver imaging features from CT scan using CNNs in CRC patients and analyze the imaging features using machine learning approaches to predict the metachronous liver metastasis. Additionally, we tried to elucidate its clinical implication in oncological perspectives using statistical analysis. As a proof of concept study, abdominal CT images were collected from 2019 patients who had colectomy for stage I-III CRC, preoperatively. 


\section{Methods}

\section{Patients and data acquisition}

We performed a retrospective, cross-sectional study in patients who underwent colectomy for CRC from January 2008 to September 2013 at Seoul National University Hospital. Stage I-III CRC patients who had curative resection were included in the analyses. The exclusion criteria consisted of patients who had surgery less than 5 years; recurrence in or distant metastasis to other than liver after in less than 5 years after surgery; preoperative neoadjuvant chemotherapy; a history of liver resection; have liver lesion; and had a poor quality of preoperative CT scan. A total of 2019 patients were eligible, and their electronic medical records (EMR) and CT images were collected. The CRC staging description was based on the AJCC staging system, seventh edition, which is a classification system provided by the American Joint Committee on Cancer for describing the extent of cancer progression [17]. We used the clinical information turned into a dichotomized form such as $\mathrm{T}$ stages into $\mathrm{T} 1$, T2 vs. T3, T4, N stages into N0 vs. N1, N2 and patient ages into $<65$ years vs. $>=65$ years. 5 -year metachronous liver metastasis (5YLM) rate was the primary endpoint. If the patient died or had recurrence other than in liver, they would not be counted as metachronous liver metastasis incidences. We used the abdominal CTs taken before the colectomy for clinical staging. For image acquisition, the non-contrast abdominal CT scan image of each patient was used. A physician (author EKC) acquired the images under the guidance of radiologist (author HSK). Cross-sectional images at the level where the caudate lob of the liver is most prominent were selected with abdominal view setting. The regions of interest (ROIs) were placed at the segment 7 of the Couinaud system [18], which is the right - posterior - superior segment of liver, with a size of $50 \times 50$ pixels.

\section{Feature generation}

Figure 1 shows the overview of the analysis framework. First, feature extraction on the images was done by utilizing a pre-trained convolutional neural network, VGG16 [19], which do not require further training. For the feature extraction, we used the fully connected layers, which is a top-layer of the pre-trained model of VGGnet with 16 layers (VGG-16). The 4096 features were extracted as an output. Then, we preprocessed the extracted imaging features rather than using whole 4096 features based on the significance of association with 5YLM rate by performing univariate logistic regression analysis. The subsets of imaging features that passed the suggestive significance level $(P<0.01)$ were used for further analyses. Lastly, principal component analysis (PCA) was performed for reducing the feature dimensionality, and this generated new sets of features, such as 1st principal component (PC1) to 10th principal component (PC10), sequentially. At the input stage to the machine learning methods, we further standardized either the clinical features or the PC-transformed image features, by $\mathrm{z}$ normalization for each feature to have a mean of zero and unit variance.

\section{Prediction models}

We trained two machine learning algorithms, including logistic regression classification (LR) and random forest classification (RFC) to predict 5YLM and compared the performances of each model. The models were designed by respective combination of features consisting of clinical features, which includes age, gender, $\mathrm{T}$ stage and $\mathrm{N}$ stage, and imaging features, which are the sequential summation of PC1 to PC10. The whole 


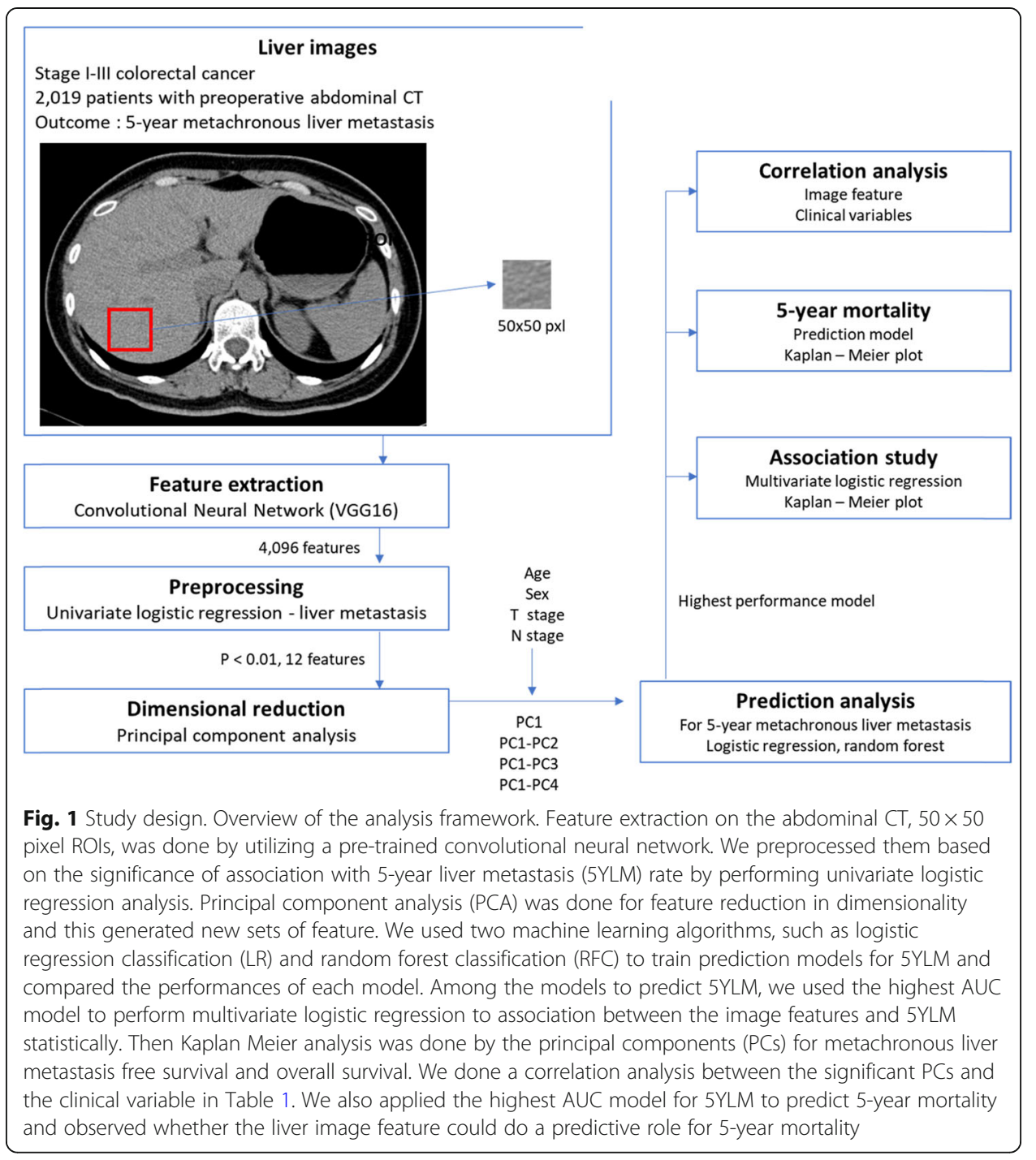

data set was divided into a training set (70\%) and a test set (30\%), and a five-fold cross validation scheme was used on the training set for the parameter tuning of the classification models. Given our highly imbalanced data set, we adopted five-fold, considering that five can be reasonable to allow enough number of positive data samples in each fold. Due to the highly imbalanced data set, we dealt with the imbalance problem of the training set first by oversampling on the negative minority class to meet negative/ positive ratio 0.2 , followed by down-sampling on the positive majority class to meet negative/positive ratio 0.4 finally. For up-sampling, we used SMOTE (Synthetic Minority Over-Sampling Technique) [20], and for down-sampling, we used the random down-sampling technique. After performing five-fold cross-validation on the training set, the trained model is validated on the test set. We note that cross-validation procedure is used to prevent overfitting to the training set when fitting the prediction model (LR and RFC). To validate the trained model, we performed the procedure aforementioned (splitting the data set into a training set and a test set; SMOTE followed by down-sampling; a five-fold cross validation to find the optimal parameters; and performance evaluation on the test set) for 100 iterations. Using the trained model, we 
evaluated the performance of the test set. The performances were measured by the area under the curve (AUC) of the receiver operating characteristics (ROC) curve and presented as means and standard deviations.

We also applied the model with the highest AUC for 5YLM to predict 5-year mortality and observed whether the liver imaging feature contribute to a prediction of 5-year mortality. The AUC performance was compared with the model using clinical feature only for the prediction of 5-year mortality.

\section{Association study}

Among the models to predict 5YLM, we used the model with the highest AUC to perform multivariate logistic regression to identify associations between the imaging features and 5YLM. Then, Kaplan-Meier analysis was conducted based on the principal components (PCs) that were significantly associated with metachronous liver metastasisfree survival and overall survival $(P<0.05)$. For survival analysis, the patients excluded by the previous exclusion criteria, such as recurrence in or distant metastasis to other than liver after in less than 5 years after surgery, were included. The optimal cut-off points for those PCs to divide patients into two groups were determined by MaxStat packages in R (Maximally selected Rank Statistics). MaxStat uses the maximally selected rank statistics to recommend the optimal cut-off point for the survival plot [21]. Univariate cox proportional hazard regression analysis was performed to compare the differences between PCbased groups using the time to event and the censoring data of it.

\section{Correlation study}

To investigate the clinical relevance of the imaging features, we ran a correlation analysis between the significant PCs and the additional clinical variables in Table 1, such as age, sex, body mass index, tumor location, alcohol consumption, liver function test, fatty liver status, $\mathrm{T}$ stage, $\mathrm{N}$ stage, Angiolymphatic invasion venous invasion, and postoperative follow-up duration. We measured Pearson's correlation between numeric variables, and Spearman's correlation between categorical variables, respectively. $P$ value of the correlation coefficient between variables less than 0.05 was considered statistically significant. The results were visualized with "corrplot" $R$ package.

All the statistical and computational analyses were done by $\mathrm{R}$ statistical software (version 3.5.3 R) and Python software (version 3.6.2). Associations between clinical information and 5YLM rate were assessed by Chi-square test, Student's t-test, and analysis of variance (ANOVA) for independent groups in Table 1.

\section{Ethics statement}

The Institutional Review Board of Seoul National University Hospital approved the study protocol (IRB number 1902-088-1010), and the study was conducted in accordance with the Declaration of Helsinki. Informed consent was waived by the board.

\section{Results}

\section{Patient demographics}

Our study sample comprised 2019 patients (1269 males and 750 females) who had colectomy for stage I-III colorectal cancer. The mean patient age was $62.32+/-9.21$ years. 
Table 1 Demographic features of the study population

\begin{tabular}{|c|c|c|c|}
\hline & $\begin{array}{l}\text { Liver metastasis, no } \\
(\boldsymbol{N}=1919,95.04 \%)\end{array}$ & $\begin{array}{l}\text { Liver Metastasis, yes } \\
(\boldsymbol{N}=100,4.96 \%)\end{array}$ & $P$ value \\
\hline Age (years) & $62.3 \pm 9.2$ & $63.1 \pm 9.5$ & 0.390 \\
\hline Age $>=65$ years & & & 0.640 \\
\hline No & $1092(56.9 \%)$ & $54(54.0 \%)$ & \\
\hline Yes & $827(43.1 \%)$ & $46(46.0 \%)$ & \\
\hline Sex & & & 0.727 \\
\hline Male & $1204(62.7 \%)$ & $65(65.0 \%)$ & \\
\hline Female & 715 (37.3\%) & $35(35.0 \%)$ & \\
\hline BMI (kg/m2) & $23.9 \pm 3.0$ & $23.8 \pm 3.1$ & 0.805 \\
\hline $\mathrm{BMI}(>=25 \mathrm{~kg} / \mathrm{m} 2)$ & & & 0.648 \\
\hline No & $1280(66.7 \%)$ & $64(64.0 \%)$ & \\
\hline Yes & $638(33.3 \%)$ & $36(36.0 \%)$ & \\
\hline Tumor location & & & 0.127 \\
\hline Right & $501(26.4 \%)$ & $19(19.0 \%)$ & \\
\hline Left & $1397(73.6 \%)$ & $81(81.0 \%)$ & \\
\hline Heavy alcohol consumption & & & 1 \\
\hline No & $1261(65.7 \%)$ & $66(66.0 \%)$ & \\
\hline Yes & $658(34.3 \%)$ & $34(34.0 \%)$ & \\
\hline GOT & $22.3 \pm 9.0$ & $21.8 \pm 9.6$ & 0.559 \\
\hline GPT & $20.3 \pm 12.8$ & $19.8 \pm 16.0$ & 0.737 \\
\hline Fatty liver & & & 0.283 \\
\hline No & $1743(95.0 \%)$ & $96(98.0 \%)$ & \\
\hline Yes & $91(5.0 \%)$ & $2(2.0 \%)$ & \\
\hline T stage & & & $<0.001$ \\
\hline T1 stage & $359(18.7 \%)$ & $4(4.0 \%)$ & \\
\hline T2 stage & $352(18.3 \%)$ & $6(6.0 \%)$ & \\
\hline T3 stage & $1106(57.6 \%)$ & $74(74.0 \%)$ & \\
\hline T4 stage & $102(5.3 \%)$ & $16(16.0 \%)$ & \\
\hline $\mathrm{N}$ stage & & & $<0.001$ \\
\hline No & $1293(67.4 \%)$ & $28(28.0 \%)$ & \\
\hline N1 & $463(24.1 \%)$ & $39(39.0 \%)$ & \\
\hline N2 & $163(8.5 \%)$ & $33(33.0 \%)$ & \\
\hline Lymph node metastasis & & & $<0.001$ \\
\hline Absent & $1293(67.4 \%)$ & $28(28.0 \%)$ & \\
\hline Present & $626(32.6 \%)$ & $72(72.0 \%)$ & \\
\hline Overall stage & & & $<0.001$ \\
\hline Stage 1 & $581(30.3 \%)$ & $6(6.0 \%)$ & \\
\hline Stage 2 & $658(34.3 \%)$ & $18(18.0 \%)$ & \\
\hline Stage 3 & $680(35.4 \%)$ & $76(76.0 \%)$ & \\
\hline Angiolymphatic invasion & & & $<0.001$ \\
\hline Absent & $1434(77.0 \%)$ & $57(57.6 \%)$ & \\
\hline Present & $429(23.0 \%)$ & $42(42.4 \%)$ & \\
\hline Venous invasion & & & $<0.001$ \\
\hline Absent & $1729(92.8 \%)$ & $75(75.8 \%)$ & \\
\hline
\end{tabular}


Table 1 Demographic features of the study population (Continued)

\begin{tabular}{llll}
\hline & $\begin{array}{l}\text { Liver metastasis, no } \\
(\boldsymbol{N}=1919,95.04 \%)\end{array}$ & $\begin{array}{l}\text { Liver Metastasis, yes } \\
(\boldsymbol{N}=100,4.96 \%)\end{array}$ & P value \\
\hline Present & $134(7.2 \%)$ & $24(24.2 \%)$ & $<0.001$ \\
Postoperative follow up duration & $1893.5 \pm 767.7$ & $1554.5 \pm 784.9$ & \\
$\begin{array}{l}\text { 5-year Mortality } \\
\text { Alive }\end{array}$ & $1919(100.0 \%)$ & $61(61.0 \%)$ & $<0.001$ \\
Dead & $0(0.0 \%)$ & $39(39.0 \%)$ & \\
\hline
\end{tabular}

There were 100 cases $(4.96 \%)$ of metachronous liver metastasis during the follow. Patient characteristics are shown in Table 1.

\section{5-year metachronous liver metastasis prediction based on principal components and} clinical information

We preprocessed the extracted imaging features from $\mathrm{CNN}$ based on the significance of association with 5YLM rate by performing univariate logistic regression analysis. Twelve features passed the suggestive significance level $(P<0.01)$. From the 12 features, using the PCA, we generated new sets of features notated as PC1 to PC10.

For 5YLM prediction models, we used PCs and clinical features as inputs to our models, in which we not only incremented the number of PCs one by one (for example, PC1, PC1-PC2, PC1-PC3 and so on), but also adopted various combinations (for example, clinical feature only model, PCs only model, clinical feature plus PC model) in order to validate which combination of features more contribute to the prediction performance. Two supervised machine learning methods, LR and RFC, were used for the performance evaluations.

The performances of each model are shown in Table 2. Model with 1st PC (PC1) showed the highest performance among other PCs combinations both in LR (mean $\mathrm{AUC}=0.606$ ) and RFC (AUC = 0.557). In the combination of clinical and imaging features, LR model trained with PC1 and clinical features showed the best performances (mean AUC = 0.747), which imply that the model using the imaging features in combination with the clinical features improved the prediction performance rather than the model using clinical feature only (mean AUC = 0.709).

\section{Association study with the oncological and clinical variables using the designed model}

For the model with PC1 and clinical features (age, sex, T stage, and N stage), which is the best performed model, an association study was done to investigate the association between the features and 5YLM. By multivariate logistic regression analysis, PC1 showed independent association with 5YLM, significantly (beta $=-3.831, P<0.001$ ) (Table 3).

For the PC1, Kaplan-Meier plots were generated for metachronous liver metastasis free survival (Fig. 2a). The patients were divided by the optimal cut offs for each PC1 based on MaxStat. (- 0.135 for the PC1 score). The results are shown in Fig. 2 with the result of univariate cox proportional hazard regression. The 5YLM-free survival of low group (PC1 score below - 0.135) was $88.7 \%$ and the high group (PC 1score above 0.135) was $95.6 \%$. We also evaluated with the Kaplan-Meier plot for overall survival 
Table 2 Performances of the prediction models in the test set for 5-year mortality and 5-year metachronous liver metastasis

\begin{tabular}{lll}
\hline Predictors & $\begin{array}{l}\text { Logistic regression classification } \\
\text { AUC (mean }+/- \text { standard deviation) }\end{array}$ & $\begin{array}{l}\text { Random forest classification } \\
\text { AUC (mean, standard deviation) }\end{array}$ \\
\hline $\begin{array}{l}\text { Prediction model for 5-year } \\
\text { metachronous liver metastasis }\end{array}$ & & \\
Clinical* & $0.709+/-0.038$ & $0.692+/-0.038$ \\
PC1 & $0.606+/-0.044$ & $0.557+/-0.043$ \\
PC1-PC2 & $0.600+/-0.042$ & $0.536+/-0.042$ \\
PC1-PC3 & $0.588+/-0.040$ & $0.503+/-0.046$ \\
PC1-PC4 & $0.580+/-0.040$ & $0.520+/-0.042$ \\
Clinical + PC1 & $0.747+/-0.036$ & $0.697+/-0.038$ \\
Clinical + PC1-PC2 & $0.744+/-0.036$ & $0.676+/-0.043$ \\
Clinical + PC1-PC3 & $0.740+/-0.038$ & $0.668+/-0.042$ \\
Clinical + PC1-PC4 & $0.736+/-0.038$ & $0.691+/-0.042$ \\
Prediction model for 5-year & & \\
mortality & & $0.679+/-0.030$ \\
Clinical* & $0.704+/-0.028$ & $0.511+/-0.030$ \\
PC1 & $0.482+/-0.031$ & $0.647+/-0.033$ \\
Clinical + PC1 & $0.695+/-0.031$ & \\
\hline
\end{tabular}

${ }^{*}$ Clinical: Age, Sex, T stage, $\mathrm{N}$ stage

and the results were similar that low group had poor overall survival compared to high group, significantly $(P<0.001)$ (Fig. $2 b)$.

In the correlation analysis between the significant PC1 and the clinical variable in Table 1, sex, body mass index, alcohol consumption and fatty liver status had significant correlation with PC1 (Fig. 3).

\section{Performance of the prediction model in 5-year mortality prediction}

We applied the 5YLM prediction model with the highest AUC model, which combined clinical features with PC1, with respect to the prediction of 5-year mortality. In the 5year mortality prediction model, the liver imaging features did not have an additional predictive power (mean AUC $=0.690$ ), when compared with clinical only models (mean AUC $=0.700$ ), in both models, trained with LR and RFC.

\section{Discussion}

Currently, clinical features, such as age, sex, $\mathrm{T}$ stage, and $\mathrm{N}$ stage are most commonly used to predict the prognosis of colorectal cancer survival [22]. Comparing the

Table 3 Multivariate logistic regression analysis for 5-year metachronous liver metastasis

\begin{tabular}{llc}
\hline & Beta (standard error) & $P$ value \\
\hline Using PCs from 12 features & & \\
Age ( $>$ 65 years) & $0.119(0.213)$ & 0.213 \\
Gender (Female) & $-0.232(0.223)$ & 0.297 \\
T3, T4 stage & $1.276(0.345)$ & $<0.001$ \\
N1, N2 stage & $1.467(0.234)$ & $<0.001$ \\
PC1 & $\mathbf{- 3 . 8 3 1 ( 1 . 0 1 2 )}$ & $\mathbf{0 . 0 0 0 1}$ \\
\hline
\end{tabular}




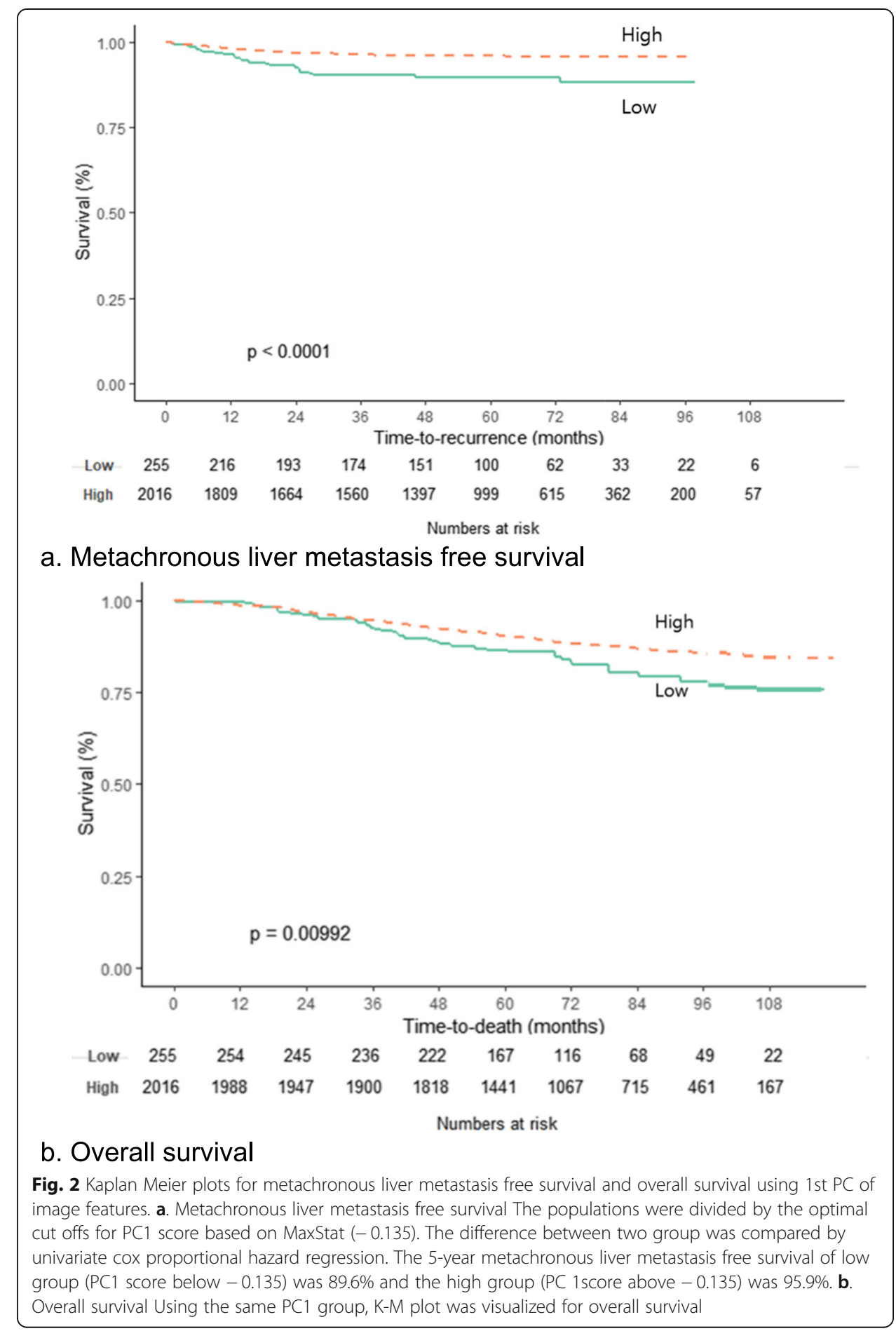

prediction performance of these clinical features, the integrative model that integrates clinical information and imaging features by convolutional neural network significantly improved performance of prediction for 5YLM rate.

This is quite interesting result because adding the $50 \times 50$ pixel liver imaging features would contain extremely limited information, but still, we demonstrated the additional imaging features when combined with clinical features improved prediction performance 


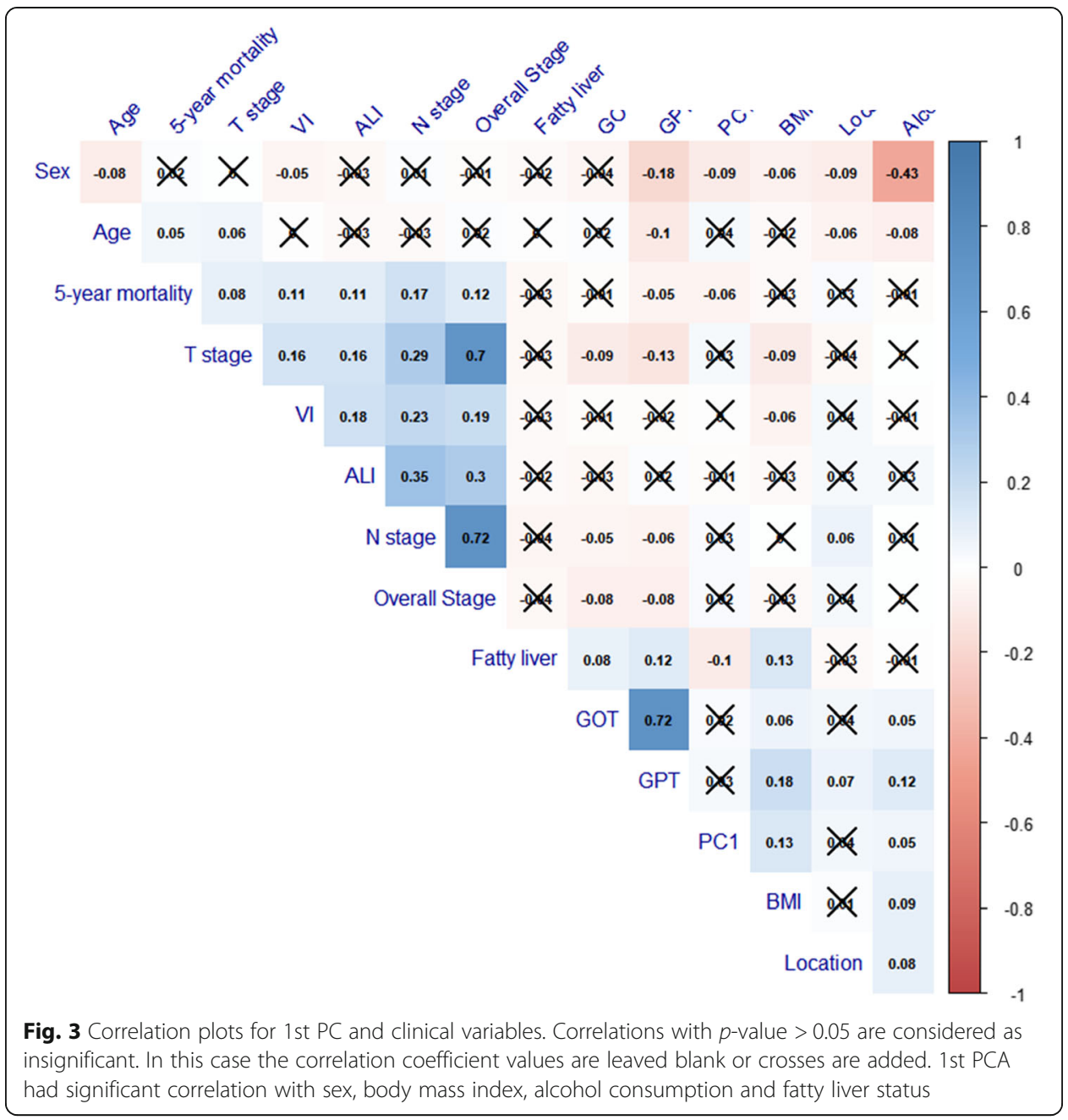

of liver metastasis. The underlying mechanism would be that, though at the time of surgery the liver does not seem to impose liver metastasis on gross finding of CT images, there could be underlying molecular changes in liver that could be predictive of liver metastasis. For 5-year mortality, the liver feature did not have additive predictive power in prediction, but there was a significant association with overall survival in K-M plot. In our study, we applied both statistical association analysis and computational predictive analysis for the outcome. Association studies would focus on understanding a relationship between the variables and outcomes, while prediction studies train the model with the training data to obtain the variables to investigate their predictive power for the corresponding outcome. Association studies might provide explanation for the relationship but might not have predictive power. On the contrary, prediction studies might have high performance but hard to interpret it. This might imply that the liver image features alone are not enough to precisely predict the outcome of 5-year mortality, however they are associated with the 5-year mortality based on the results of Kaplan-Meier survival analysis with significant $p$-values.

Metachronous liver metastasis significantly influence the prognosis of CRC patients who had curative colectomy $[16,23]$, and it is reported that in $20-30 \%$ of patients, it 
will be detected following primary colectomy [24-26]. Well-known risk factors for liver metastasis are $\mathrm{N}$ stage, vascular invasion and preoperative carcinoembryonic antigen (CEA) level [23, 27, 28]. Additionally, there are also suggested factors predisposing to the development of metachronous liver metastasis in CRC, such as tissue microenvironmental changes and chronic inflammation [29]. In the present study, we focused on investigating the clinical relevance of this imaging features performing association and correlation studies with patient's demographical and oncological information. The representative imaging feature, the 1st principal component (PC1), had a significant association with 5YLM-free survival, and the results were shown in Kaplan-Meier plot. In the correlation study, PC1 had significant correlations with sex, body mass index, alcohol consumption and fatty liver status. Sex [30], body mass index [29], alcohol consumption [31] and fatty liver status [32] are reported to be correlated with liver metastasis in CRC. Obesity determined by body mass index change or sex difference and hepatosteatosis derivative of fatty liver or alcoholism are suggestive predisposing factors in the function of tissue microenvironment change and chronic inflammation [29]. It can be postulated that the imaging features of liver ROIs impose various heterogeneity of predisposing factors for metachronous liver metastasis comprehensively.

In the study design, we additionally preprocessed the 4096 imaging features extracted by training the convolutional neural network, VGG16, based on the significance of association with 5YLM rate. VGG16 is a convolutional neural network model which achieved 92,7\% accuracy in ImageNet, a dataset of over 14 million images belonging to 1000 classes, and it used RGB images for training [19]. This model can capture complex features like human faces, natural scenes and showed human level performance [33]. The preprocessing step was introduced because designing the prediction model with the whole 4096 imaging features from CNN did not provide good results for 5YLM (data not shown). This might come from the fact that pretrained CNN basically trained RGB images which has chromatic color, shape and size variance but in CT scan image, it is achromatic color, fixed square shape and fixed $50 \times 50$ pixel sized. Thus, preprocessing with statistical association threshold will remove the noises and include only the effective features to be introduced.

With the preprocess features, principal component analysis was performed for reducing the feature dimensionality, and new sets of features, such as 1st principal component $(\mathrm{PC1})$ to 10th principal component (PC10) as an imaging features for the prediction model and showed that $\mathrm{PC} 1$ had improved the performance of prediction model by clinical information to predict 5YLM. We used the PC transformed as imaging features rather than the ones after preprocessing, which is primarily generated by $\mathrm{CNN}$, because these did not have promising results in the prediction model (data not shown). This might reflect the fact that the respective imaging feature by itself do not have meaningful contribution for prediction but the selected features should be aggregated to have a predictive role in the model.

In our study, we used logistic regression and random forest classification, which are very common and widely studied machine learning models [34]. For the prediction of 5YLM, the model trained by logistic regression showed the improvement by adding imaging features to the clinical features compared with the ones only consisting of clinical features. However, the improvement of performances was modest by the models trained by random forest classification. Logistic regression is a classical machine 
learning classifier and it has advantage of having fast speed to train the inputs and making it more interpretable [6,35]. In a recent study, comparing logistic regression and random forest classification for binary outcomes, when increasing the variance in the explanatory and noise variables, logistic regression consistently better performed as compared to random forest classification [34]. However, true positive rate was higher in random forest classification compared to logistic regression. When machine learning is applied to the clinical fields, the characteristics of the data set and the purpose of using machine learning should be considered carefully before choosing which algorithms to be applied.

This study has several advantages. First, we comprehensively analyzed the imaging feature by respective combination of image features and clinical information. By this way, we were able to find that the best-performing combination of imaging features, preprocessed features by association analysis and PCs from PCA analysis, which showed improved prediction performance combined with clinical information. Second, we performed both the prediction model based on computational analysis and association and correlation study based on statistical analysis. This will help to interpret the complex nature of the liver CT scan image features more intuitively, provide the clinical relevance of it and support an evidence for the results of prediction model. Third, all the patients enrolled in the analyses had at least 5 years of follow-up after primary colectomy. This will make the results of analyses more reliable since the information involves the long-term observations.

Despite demonstrating the validity of our proposed approach, there might be a couple of remaining potential limitations. First, as we used the retrospectively collected CT scan, we could not collect the parameters and the product information of the CT scanner, which could influence the imaging features as a batch effect. But since, the CT images were taken on the purpose of preoperative clinical staging in colorectal cancer, the quality of the CT images was well controlled. Second, we simply concatenated the clinical factors and imaging features level for integration. Transformational integration could be applied in a larger set of samples [36]. Third, since there are no open source databases that includes both abdominal CT images and clinical information, we could not replicate the results in another data set.

\section{Conclusion}

By analyzing the liver image features by convolutional neural network in stage I-III colorectal cancer patients based on preoperative abdominal CT scan, we were able identify the contribution of imaging features to predict the metachronous liver metastasis. The 1st PC from imaging feature combined with clinical information improved the performance of standardized prediction model using only clinical information such as age and TNM stage. The preoperative liver imaging features generated by CNN might have the potential to predict liver metastasis after 5 years. This result suggests that even though there were no metastasis or liver lesion during the primary colectomy for stage I-III CRC, the features of liver in preoperative CT scan can impose characteristics that could be predictive for liver metastasis during the postoperative follow-up. The findings might conceptualize the importance of the imaging features in liver which could be applied for clinical practice. 


\section{Abbreviations}

CRC: Colorectal cancer; CNN: Convolutional neural network; 5YLM: 5-year metachronous liver metastasis; PCs: Principal components; AUC: Area under the curved; VGG-16: VGGnet with 16 layers; PCA: Principal component analysis; PC1: 1st principal component; PC10: 10th principal component; LR: Logistic regression; RFC: Random forest classification; SMOTE: Synthetic Minority Over-Sampling Technique

\section{About this supplement}

This article has been published as part of BMC Bioinformatics Volume 21 Supplement 13, 2020: Selected articles from the 18th Asia Pacific Bioinformatics Conference (APBC 2020): bioinformatics. The full contents of the supplement are available online at https://bmcbioinformatics.biomedcentral.com/articles/supplements/volume-21-supplement-13.

\section{Authors' contributions}

SL, EKC, KJP, DK conceptualized and designed the study; EKC, KJP provided the study materials and patients; SL, EKC, SYK, KJP, DK analyzed and interpreted the patient data regarding the colorectal cancer; EK, HSK performed evaluation of the data quality and processing the raw data set; and SL, EKC, KJP, DK were a major contributor in writing the manuscript. All authors read and approved the final manuscript and are accountable for all aspects of the work.

\section{Funding}

The support for this research in the design of the study, and analysis, interpretation of data and in writing the manuscript was provided by NLM R01 LM012535. Publication costs are funded by NLM R01 funding (LM012535).

\section{Availability of data and materials}

The datasets generated and/or analyzed during the current study are not publicly available due to restrictions (the institution policy to protect the privacy of research participants) but are available from the corresponding author on reasonable request.

\section{Ethics approval and consent to participate}

The Institutional Review Board of Seoul National University Hospital approved the study protocol (IRB number 1902088-1010), and the study was conducted in accordance with the Declaration of Helsinki. Informed consent was waived by the board.

\section{Consent for publication}

Not applicable.

\section{Competing interests}

The authors declare that they have no competing interests.

\section{Author details}

'Division of Future Convergent, The Cyber University of Korea, Seoul 03051, South Korea. ${ }^{2}$ Department of Surgery, Seoul National University Hospital Healthcare System Gangnam Center, Seoul 06236, South Korea. ${ }^{3}$ Department of Biostatistics, Epidemiology \& Informatics, Perelman School of Medicine, University of Pennsylvania, B304 Richards Building, 3700 Hamilton Walk, Philadelphia, PA 19104-6116, USA. ${ }^{4}$ Department of Software and Computer Engineering, Ajou University, Suwon 16499, South Korea. ${ }^{5}$ Department of Radiology, Seoul National University College of Medicine, Seoul 03080, South Korea. ${ }^{6}$ Department of Surgery, Seoul National University College of Medicine, Seoul 03080, South Korea. ${ }^{7}$ Institute for Biomedical Informatics, University of Pennsylvania, Philadelphia, PA 19104, USA.

\section{Published: 17 September 2020}

\section{References}

1. Sheth KR, Clary BM. Management of hepatic metastases from colorectal cancer. Clin Colon Rectal Surg. 2005; 18(3):215-23.

2. Garden OJ, Rees M, Poston GJ, Mirza D, Saunders M, Ledermann J, et al. Guidelines for resection of colorectal cancer liver metastases. Gut. 2006;55(Suppl 3):iii1-8.

3. Hadden WJ, de Reuver PR, Brown K, Mittal A, Samra JS, Hugh TJ. Resection of colorectal liver metastases and extra-hepatic disease: a systematic review and proportional meta-analysis of survival outcomes. HPB (Oxford). 2016;18(3):209-20.

4. Valderrama-Trevino Al, Barrera-Mera B, Ceballos-Villalva JC, Montalvo-Jave EE. Hepatic metastasis from colorectal cancer Euroasian J Hepatogastroenterol. 2017;7(2):166-75.

5. Choi ER, Lee HY, Jeong JY, Choi YL, Kim J, Bae J, et al. Quantitative image variables reflect the intratumoral pathologic heterogeneity of lung adenocarcinoma. Oncotarget. 2016;7(41):67302-13.

6. Forghani R, Savadjiev P, Chatterjee A, Muthukrishnan N, Reinhold C, Forghani B. Radiomics and artificial intelligence for biomarker and prediction model development in oncology. Comput Struct Biotechnol J. 2019;17:995-1008.

7. Litjens $\mathrm{G}$, Kooi T, Bejnordi BE, Setio AAA, Ciompi F, Ghafoorian M, et al. A survey on deep learning in medical image analysis. Med Image Anal. 2017:42:60-88.

8. Meng Y, Zhang Y, Dong D, Li C, Liang X, Zhang C, et al. Novel radiomic signature as a prognostic biomarker for locally advanced rectal cancer. J Magn Reson Imaging. 2018;48(3):605

9. Li Q, Kim J, Balagurunathan Y, Liu Y, Latifi K, Stringfield O, et al. Imaging features from pretreatment CT scans are associated with clinical outcomes in nonsmall-cell lung cancer patients treated with stereotactic body radiotherapy. Med Phys. 2017;44(8):4341-9. 
10. Lubner MG, Stabo N, Lubner SJ, del Rio AM, Song C, Halberg RB, et al. CT textural analysis of hepatic metastatic colorectal cancer: pre-treatment tumor heterogeneity correlates with pathology and clinical outcomes. Abdom Imaging. 2015;40(7):2331-7.

11. Wu W, Parmar C, Grossmann P, Quackenbush J, Lambin P, Bussink J, et al. Exploratory study to identify Radiomics classifiers for lung cancer histology. Front Oncol. 2016;6:71.

12. Chamming's F, Ueno Y, Ferre R, Kao E, Jannot AS, Chong J, et al. Features from computerized texture analysis of breast cancers at pretreatment MR imaging are associated with response to neoadjuvant chemotherapy. Radiology. 2018; 286(2):412-20

13. Kickingereder P, Burth S, Wick A, Gotz M, Eidel O, Schlemmer HP, et al. Radiomic profiling of glioblastoma: identifying an imaging predictor of patient survival with improved performance over established clinical and radiologic risk models. Radiology. 2016;280(3):880-9.

14. Deo RC. Machine learning in medicine. Circulation. 2015;132(20):1920-30.

15. Krupinski EA. Current perspectives in medical image perception. Atten Percept Psychophysiol. 2010;72(5):1205-17.

16. Sendak M, Gao M, Nichols M, Lin A, Balu S. Machine learning in health care: a critical appraisal of challenges and opportunities. EGEMS (Wash DC). 2019;7(1):1.

17. Edge SB, Compton CC. The American joint committee on cancer: the 7th edition of the AJCC cancer staging manual and the future of TNM. Ann Surg Oncol. 2010:17(6):1471-4.

18. Couinaud C. Liver lobes and segments: notes on the anatomical architecture and surgery of the liver. Presse Med. 1954; 62(33):709-12

19. Simonyan K, Zisserman A. Very deep convolutional networks for large-scale image recognition. ICLR. 2015;2015:1-14.

20. Nakamura M, Kajiwara Y, Otsuka A, Kimura H. LVQ-SMOTE - learning vector quantization based synthetic minority oversampling technique for biomedical data. BioData Min. 2013;6(1):16.

21. Hothorn T, Lausen B. On the exact distribution of maximally selected rank statistics. J Comput Stat Data Anals. 2003; 43(2):121-37.

22. Oliveira T, Silva A, Satoh K, Julian V, Leao P, Novais P. Survivability prediction of colorectal cancer patients: a system with evolving features for continuous improvement. Sensors (Basel). 2018;18:9.

23. Assumpcao L, Choti MA, Gleisner AL, Schulick RD, Swartz M, Herman J, et al. Patterns of recurrence following liver resection for colorectal metastases: effect of primary rectal tumor site. Arch Surg. 2008:143(8):743-9 discussion 749-750.

24. Fegiz G, Ramacciato G, D'Angelo F, Barillari P, Indinnimeo M, Gozzo P, et al. Patient selection and factors affecting results following resection for hepatic metastases from colorectal carcinoma. Int Surg. 1991;76(1):58-63.

25. Abdalla EK. Resection of colorectal liver metastases. J Gastrointest Surg. 2011;15(3):416-9.

26. Scheele J, Altendorf-Hofmann A. Resection of colorectal liver metastases. Langenbeck's Arch Surg. 1999;384(4):313-27.

27. Cromheecke M, de Jong KP, Hoekstra HJ. Current treatment for colorectal cancer metastatic to the liver. Eur J Surg Oncol. 1999;25(5):451-63.

28. Minsky B, Mies C. The clinical significance of vascular invasion in colorectal cancer. Dis Colon Rectum. 1989;32(9):794-803.

29. Pathak S, Pandanaboyana S, Daniels I, Smart N, Prasad KR. Obesity and colorectal liver metastases: mechanisms and management. Surg Oncol. 2016;25(3):246-51.

30. Sorski L, Levi B, Shaashua L, Neeman E, Benish M, Matzner P, et al. Impact of surgical extent and sex on the hepatic metastasis of colon cancer. Surg Today. 2014;44(10):1925-34

31. Mohr AM, Gould JJ, Kubik JL, Talmon GA, Casey CA, Thomas P, et al. Enhanced colorectal cancer metastases in the alcohol-injured liver. Clin Exp Metastasis. 2017;34(2):171-84.

32. Wu K, Zhai MZ, Weltzien EK, Cespedes Feliciano EM, Meyerhardt JA, Giovannucci E, et al. Non-alcoholic fatty liver disease and colorectal cancer survival. Cancer Causes Control. 2019;30(2):165-8.

33. Xu Y, Jia Z, Wang LB, Ai Y, Zhang F, Lai M, et al. Large scale tissue histopathology image classification, segmentation, and visualization via deep convolutional activation features. BMC Bioinformatics. 2017;18(1):281.

34. Kirasich K, Smith T, Sadler B. Random Forest vs logistic regression: binary classification for heterogeneous datasets. SMU Data Sci Rev. 2018;1(3):1-24.

35. Christodoulou E, Ma J, Collins GS, Steyerberg EW, Verbakel JY, Van Calster B. A systematic review shows no performance benefit of machine learning over logistic regression for clinical prediction models. J Clin Epidemiol. 2019;110:12-22.

36. Ritchie MD, Holzinger ER, Li R, Pendergrass SA, Kim D. Methods of integrating data to uncover genotype-phenotype interactions. Nat Rev Genet. 2015;16(2):85-97.

\section{Publisher's Note}

Springer Nature remains neutral with regard to jurisdictional claims in published maps and institutional affiliations.

Ready to submit your research? Choose BMC and benefit from:
- fast, convenient online submission
- thorough peer review by experienced researchers in your field
- rapid publication on acceptance
- support for research data, including large and complex data types
- gold Open Access which fosters wider collaboration and increased citations
- maximum visibility for your research: over 100M website views per year
At BMC, research is always in progress.
Learn more biomedcentral.com/submissions

